\title{
TEMPORAL DICTIONARY LEARNING FOR TIME-SERIES DECOMPOSITION
}

\section{Jens Bürger and Jorge Calvimontes}

\begin{abstract}
Dictionary Learning (DL) is a feature learning method that derives a finite collection of dictionary elements (atoms) from a given dataset. These atoms are small characteristic features representing recurring patterns within the data. A dictionary therefore is a compact representation of complex or large scale datasets. In this paper we investigate DL for temporal signal decomposition and reconstruction. Decomposition is a common method in time-series forecasting to separate a complex composite signal into different frequency components as to reduce forecasting complexity. By representing characteristic features, we consider dictionary elements to function as filters for the decomposition of temporal signals. Rather than simple filters with clearly defined frequency spectra, we hypothesize for dictionaries and the corresponding reconstructions to act as more complex filters. Training different dictionaries then permits to decompose the original signal into different components. This makes it a potential alternative to existing decomposition methods. We apply a known sparse DL algorithm to a wind speed dataset and investigate decomposition quality and filtering characteristics. Reconstruction accuracy serves as a proxy for evaluating the dictionary quality and a coherence analysis is performed to analyze how different dictionary configurations lead to different filtering characteristics. The results of the presented work demonstrate how learned features of different dictionaries represent transfer functions corresponding to frequency components found in the original data. Based on finite sets of atoms, dictionaries provide a deterministic mechanism to decompose a signal into various reconstructions and their respective remainders. These insights have direct application to the investigation and development of advanced signal decomposition and forecasting techniques.
\end{abstract}

Keywords: Dictionary Learning, SAILnet, Time-Series, Decomposition.

DOI: 10.23881/idupbo.019.1-7i 